\title{
Yosida approximation equations technique for system of generalized set-valued variational inclusions
}

\section{Han-Wen Cao*}

${ }^{*}$ Correspondence:

chwhappy@163.com

Department of Science, Nanchang

Institute of Technology, Nanchang,

330099, P.R. China

\begin{abstract}
In this paper, under the assumption with no continuousness, a new system of generalized variational inclusions in the Banach space is introduced. By using the Yosida approximation operator technique, the existence and uniqueness theorems for solving this kind of variational inclusion are established.

MSC: $49 \mathrm{H} 09 ; 49 \mathrm{~J} 40 ; 49 \mathrm{H} 10$

Keywords: system of generalized variational inclusions; $m$-accretive mapping; resolvent operators; approximation operators
\end{abstract}

\section{Introduction}

Variational inclusions are useful and important extensions and generalizations of the variational inequalities with a wide range of applications in industry, mathematical finance, economics, decisions sciences, ecology, mathematical and engineering sciences. In general, the method based on the resolvent operator technique has been widely used to solve variational inclusions.

In this paper, under the assumption with no continuousness, we first introduce a new system of generalized variational inclusions in the Banach space. By using the Yosida approximation technique for $m$-accretive operator, we prove some existence and uniqueness theorems of solutions for this kind of system of generalized variational inclusions. Our results generalize and improve main results in [1-7].

For $i=1,2$, let $E_{i}$ be a real Banach space, let $T_{i}: E_{i} \rightarrow 2^{E_{i}}, M_{i}: E_{1} \times E_{2} \rightarrow 2^{E_{i}}$ be setvalued mappings, let $h_{i}, g_{i}: E_{i} \rightarrow E_{i}, F_{i}: E_{1} \times E_{2} \rightarrow E_{i}$ be single-valued mappings, and let $\left(f_{1}, f_{2}\right) \in E_{1} \times E_{2}$. We consider the following problem: finding $(x, y) \in E_{1} \times E_{2}$ such that

$$
\left\{\begin{array}{l}
f_{1} \in T_{1} x+F_{1}(x, y)+M_{1}\left(h_{1}(x), g_{1}(x)\right) ; \\
f_{2} \in T_{2} y+F_{2}(x, y)+M_{2}\left(h_{2}(y), g_{2}(y)\right) .
\end{array}\right.
$$

This problem is called the system of generalized set-valued variational inclusions.

There are some special cases in literature.

(2013 Cao; licensee Springer. This is an Open Access article distributed under the terms of the Creative Commons Attribution License (http://creativecommons.org/licenses/by/2.0), which permits unrestricted use, distribution, and reproduction in any medium, provided the original work is properly cited. 
(1) If $T_{1}=0, T_{2}=0, f_{1}=0, f_{2}=0$, then (1.1) reduces to the problem of finding $(x, y) \in$ $E_{1} \times E_{2}$ such that

$$
\left\{\begin{array}{l}
0 \in F_{1}(x, y)+M_{1}\left(h_{1}(x), g_{1}(x)\right) \\
0 \in F_{2}(x, y)+M_{2}\left(h_{2}(y), g_{2}(y)\right) .
\end{array}\right.
$$

Problem (1.2) was introduced and studied by Kazmi and Khan $[1,2]\left(g_{1}=g_{2}=I\right.$ in [2]).

(2) If $h_{i}=g_{i}=I$ is the identity operator, $M_{i}(\cdot, \cdot)=0, f_{1}=f_{2}=0$, then (1.1) reduces to the problem of finding $(x, y) \in E_{1} \times E_{2}$ such that

$$
\left\{\begin{array}{l}
0 \in T_{1} x+F_{1}(x, y) \\
0 \in T_{2} y+F_{2}(x, y) .
\end{array}\right.
$$

Problem (1.3) was introduced and studied by Verma [3], Fang and Huang [5].

(3) If $E_{1}=E_{2}=H$ is a Hilbert space, $F_{1}=F_{2}=F(x), M(\cdot, \cdot)=M(\cdot), f_{1}=f_{2}=0$, then (1.1) reduces to the problem of finding $x \in H$ such that

$$
0 \in F(x)+M(x)
$$

Problem (1.4) was introduced and studied by Zeng et al. [6]. If $F(x)=S(x)-T(x)-f, f \neq 0$, (1.4) becomes $f \in S(x)-T(x)+M(x)$ considered by Verma [4].

Let $E$ be a real Banach space with dual $E^{*}, J: E \rightarrow 2^{E^{*}}$ is the normalized duality mapping defined by

$$
J x=\left\{f \in E^{*}:\langle x, f\rangle=\|x\|^{2}=\|f\|^{2}\right\}
$$

where $\langle\cdot, \cdot\rangle$ denotes the generalized duality paring. In the sequel, we shall denote the singlevalued normalized duality map by $j$. It is well known that if $E$ is smooth, then $J$ is singlevalued, and $E^{*}$ is uniformly convex, then $j$ is uniformly continuous on bounded set.

We assume that $E, E_{1}, E_{2}$ are smooth Banach spaces. For convenience, the norms of $E$, $E_{1}$ and $E_{2}$ are all denoted by $\|\cdot\|$. The norm of $E_{1} \times E_{2}$ is defined by $\|\cdot\|+\|\cdot\|$, i.e., if $(x, y) \in E_{1} \times E_{2}$, then $\|(x, y)\|=\|x\|+\|y\|$.

Definition 1.1 Let $T: E \rightarrow 2^{E}$ be a set-valued mapping.

(i) $T$ is said to be accretive, if $\forall x, y \in E, u \in T x, v \in T y$,

$$
\langle u-v, j(x-y)| \geq 0, \quad \forall x, y \in E, u \in T x, v \in T y .
$$

(ii) $T$ is said to be $\alpha$-strongly-accretive if there exists $\alpha>0$ such that $\forall x, y \in E, u \in T x$, $v \in T y$,

$$
\langle u-v, j(x-y)\rangle \geq \alpha\|x-y\|^{2} .
$$

(iii) $T$ is said to be $m$-accretive if $T$ is accretive and $(I+\lambda T)(E)=E, \forall \lambda>0$. 
Definition 1.2 Let $N: E_{1} \times E_{2} \rightarrow 2^{E_{1}}$ be a set-valued mapping.

(i) The mapping $x \mapsto N(x, y)$ is said to be accretive if $\forall x_{1}, x_{2} \in E_{1}, u \in N\left(x_{1}, y\right)$, $v \in N\left(x_{2}, y\right), y \in E_{2}$,

$$
\left\langle u-v, j\left(x_{1}-x_{2}\right)\right\rangle \geq 0
$$

(ii) The mapping $x \mapsto N(x, y)$ is said to be $\alpha$-strongly-accretive if there exists $\alpha>0$ such that $\forall x_{1}, x_{2} \in E_{1}, u_{1} \in N\left(x_{1}, y\right), u_{2} \in N\left(x_{2}, y\right), y \in E_{2}$,

$$
\left\langle u_{1}-u_{2}, j\left(x_{1}-x_{2}\right)\right\rangle \geq \alpha\left\|x_{1}-x_{2}\right\|^{2} .
$$

(iii) The mapping $x \mapsto N(x, y)$ is said to be $m$ - $\alpha$-strongly-accretive if $N(\cdot, y)$ is $\alpha$-strongly-accretive and $(I+N(\cdot, y))(E)=E, \forall y \in E_{2}, \lambda>0$.

In a similar way, we can define the strong accretiveness of the mapping $N: E_{1} \times E_{2} \rightarrow 2^{E_{2}}$ with respect to the second argument.

Definition 1.3 Let $T: E \rightarrow 2^{E}$ be $m$-accretive mapping.

(i) The resolvent operator of $T$ is defined by $R_{\lambda}^{T} x=(I+\lambda T)^{-1} x, \forall x \in E, \lambda>0$.

(ii) The Yosida approximation of $T$ is defined by $J_{\lambda}^{T} x=\frac{1}{\lambda}\left(I-R_{\lambda}^{T}\right) x, \forall x \in E, \lambda>0$.

Definition 1.4 The mapping $F: E_{1} \times E_{2} \rightarrow E$ is said to be $(r, s)$-mixed Lipschitz continuous if there exist $r>0, s>0$ such that $\forall\left(x_{1}, y_{1}\right),\left(x_{2}, y_{2}\right) \in E_{1} \times E_{2}$,

$$
\left\|F\left(x_{1}, y_{1}\right)-F\left(x_{2}, y_{2}\right)\right\| \leq r\left\|x_{1}-x_{2}\right\|+s\left\|y_{1}-y_{2}\right\| .
$$

In the sequel, we use the notation $\rightarrow$ and $\rightarrow$ to denote strong and weak convergence, respectively.

Proposition 1.1 [8-10] If $T: E \rightarrow 2^{E}$ is m-accretive, then

(1) $R_{\lambda}^{T}$ is single-valued and $\left\|R_{\lambda}^{T} x-R_{\lambda}^{T} y\right\| \leq\|x-y\|, \forall x, y \in E$;

(2) $\left\|J_{\lambda}^{T} x\right\| \leq|T x|=\inf \{\|y\|: y \in T x\}, \forall x \in D(T)$;

(3) $J_{\lambda}^{T}$ is m-accretive on $E$, and $\left\|J_{\lambda}^{T} x-J_{\lambda}^{T} y\right\| \leq \frac{2}{\lambda}\|x-y\|, \forall x, y \in E, \lambda>0$;

(4) $J_{\lambda}^{T} x \in T R_{\lambda}^{T} x$;

(5) If $E^{*}$ is uniformly convex Banach space, then $T$ is demiclosed, i.e., $\left[x_{n}, y_{n}\right] \in \operatorname{Graph}(T), x_{n} \rightarrow x, y_{n} \rightarrow y$ implies that $[x, y] \in \operatorname{Graph}(T)$.

Lemma 1.1 If $T: E \rightarrow 2^{E}$ is $m$ - $\alpha$-strongly-accretive, then

(i) $R_{\lambda}^{T}$ is $\frac{1}{1+\lambda \alpha}$-Lipschitz continuous;

(ii) $J_{\lambda}^{T}$ is $\frac{\alpha}{1+\lambda \alpha}$-strongly-accretive.

Proof (i) Let $u=R_{\lambda}^{T} x, v=R_{\lambda}^{T} y$. Then $x-u \in \lambda T u, y-v \in \lambda T v$. Since $T$ is $\alpha$-stronglyaccretive, $\lambda T$ is $\lambda \alpha$-strongly-accretive, $\lambda \alpha\|u-v\|^{2} \leq\langle x-u-y+v, j(u-v)\rangle \leq\|x-y\| \| u-$ $v\|-\| u-v \|^{2}$. Therefore, $\|u-v\| \leq \frac{1}{1+\lambda \alpha}\|x-y\|$. This completes the proof of (i). 
(ii) By definition of $J_{\lambda}^{T}$ and (i), we have

$$
\begin{aligned}
\left\langle J_{\lambda}^{T} x-J_{\lambda}^{T} y, j(x-y)\right\rangle & =\frac{1}{\lambda}\left\langle x-y-\left(R_{\lambda}^{T} x-R_{\lambda}^{T} y\right), j(x-y)\right\rangle \\
& \geq \frac{1}{\lambda}\left(\|x-y\|^{2}-\left\|R_{\lambda}^{T} x-R_{\lambda}^{T} y\right\|\|x-y\|\right) \geq \frac{\alpha}{1+\lambda \alpha}\|x-y\|^{2} .
\end{aligned}
$$

This completes the proof of (ii).

Remark 1.1 Let $N_{i}: E_{1} \times E_{2} \rightarrow 2^{E_{i}}$ be set-valued mapping, let $x \mapsto N_{1}(x, y)$ and $y \mapsto$ $N_{2}(x, y)$ be $m$-accretive. Then the resolvent operator and Yosida approximation of $N_{i}$ can be rewritten as

$$
\begin{array}{ll}
R_{\lambda}^{N_{1}(\cdot, y)} x=\left(I+\lambda N_{1}(\cdot, y)\right)^{-1} x, & J_{\lambda}^{N_{1}(\cdot, y)} x=\frac{1}{\lambda}\left(I-R_{\lambda}^{N_{1}(\cdot, y)}\right) x, \\
R_{\lambda}^{N_{2}(x, \cdot)} y=\left(I+\lambda N_{2}(x, \cdot)\right)^{-1} y, & J_{\lambda}^{N_{2}(x, \cdot)} y=\frac{1}{\lambda}\left(I-R_{\lambda}^{N_{2}(x, \cdot)}\right) y,
\end{array}
$$

respectively.

Lemma 1.2 Let $N_{1}(x, y)=T_{1} x+F_{1}(x, y)$ and $N_{2}(x, y)=T_{2} y+F_{2}(x, y)$. If $T_{i}: E_{i} \rightarrow 2^{E_{i}}$ is maccretive, $F_{i}: E_{1} \times E_{2} \rightarrow E_{i}$ is $\alpha_{i}$-strongly-accretive in the ith argument, and $\left(r_{i}, s_{i}\right)$-mixed Lipschitz continuous, then

(i) $N_{i}$ is $m$ - $\alpha_{i}$-strongly-accretive in the ith argument $(i=1,2)$;

(ii) $\left\|R_{\lambda}^{N_{1}\left(\cdot, y_{1}\right)} x-R_{\lambda}^{N_{1}\left(\cdot, y_{2}\right)} x\right\| \leq \lambda s_{1}\left\|y_{1}-y_{2}\right\|$;

(iii) $\left\|R_{\lambda}^{N_{2}\left(x_{1}, \cdot\right)} y-R_{\lambda}^{N_{2}\left(x_{2}, \cdot\right)} y\right\| \leq \lambda r_{2}\left\|x_{1}-x_{2}\right\|$.

Proof (i) The fact directly follows from Kobayashi [11] (Theorem 5.3).

(ii) Let $u=R_{\lambda}^{N_{1}\left(\cdot, y_{1}\right)} x, v=R_{\lambda}^{N_{1}\left(\cdot, y_{2}\right)} x$. Then

$$
x-u-\lambda F_{1}\left(u, y_{1}\right) \in \lambda T_{1} u, \quad x-v-\lambda F_{1}\left(v, y_{2}\right) \in \lambda T_{1} v .
$$

By accretiveness of $T_{i}$ and $\alpha_{i}$-strong accretiveness of $F_{i}$, we have that

$$
\begin{aligned}
0 & \leq\left\langle-u-\lambda F_{1}\left(u, y_{1}\right)+v+\lambda F_{1}\left(v, y_{2}\right), j(u-v)\right\rangle \\
& =-\|u-v\|^{2}+\lambda\left\langle F_{1}\left(v, y_{2}\right)-F_{1}\left(u, y_{1}\right), j(u-v)\right\rangle \\
& =-\|u-v\|^{2}+\lambda\left\langle F_{1}\left(v, y_{2}\right)-F_{1}\left(u, y_{2}\right), j(u-v)\right\rangle+\lambda\left\langle F_{1}\left(u, y_{2}\right)-F_{1}\left(u, y_{1}\right), j(u-v)\right\rangle \\
& \leq-\|u-v\|^{2}-\lambda \alpha_{1}\|u-v\|^{2}+\lambda\left\|F_{1}\left(u, y_{2}\right)-F_{1}\left(u, y_{1}\right)\right\|\|u-v\| \\
& \leq-\left(1+\lambda \alpha_{1}\right)\|u-v\|^{2}+\lambda s_{1}\left\|y_{1}-y_{2}\right\|\|u-v\|,
\end{aligned}
$$

Therefore, $\|u-v\| \leq \frac{\lambda s_{1}}{1+\lambda \alpha_{1}}\left\|y_{1}-y_{2}\right\| \leq \lambda s_{1}\left\|y_{1}-y_{2}\right\|$. This completes the proof of (ii).

(iii) The proof is similar. We omit it.

\section{Main results}

We assume that $\mathrm{CB}(E)$ in the family of all nonempty closed and bounded subset of $E$. 
Lemma 2.1 [12] Let $T_{1}: E_{1} \times E_{2} \rightarrow E_{1}$ and $T_{2}=E_{1} \times E_{2} \rightarrow E_{2}$ be two continuous mappings. If there exist $\theta_{1}, \theta_{2}, 0<\theta_{1}, \theta_{2}<1$ such that

$$
\begin{aligned}
& \left\|T_{1}\left(x_{1}, y_{1}\right)-T_{1}\left(x_{2}, y_{2}\right)\right\|+\left\|T_{2}\left(x_{1}, y_{1}\right)-T_{2}\left(x_{2}, y_{2}\right)\right\| \\
& \quad \leq \theta_{1}\left\|x_{1}-x_{2}\right\|+\theta_{2}\left\|y_{1}-y_{2}\right\|,
\end{aligned}
$$

then there exists $(x, y) \in E_{1} \times E_{2}$ such that $x=T_{1}(x, y), y=T_{2}(x, y)$.

Theorem 2.1 For $i=1,2$, let $E_{i}$ be a real Banach space with uniformly convex dual $E_{i}^{*}$, and let $F_{i}: E_{1} \times E_{2} \rightarrow E_{i}, h_{i}, g_{i}: E_{i} \rightarrow E_{i}$ be three single-valued mappings, let $T_{i}: E_{i} \rightarrow 2^{E_{i}}$, $M_{i}: E_{i} \times E_{i} \rightarrow 2^{E_{i}}$ be two set-valued mappings satisfying the following conditions that

(1) $M_{i}\left(h_{i}(\cdot), g_{i}(\cdot)\right): E_{i} \rightarrow \mathrm{CB}\left(E_{i}\right)$ is m-accretive;

(2) $T_{i}$ is $m$-accretive.

(3) $F_{i}$ is $\alpha_{i}$-strongly-accretive in the ith argument and $\left(r_{i}, s_{i}\right)$-mixed Lipschitz continuous, $N_{1}(x, y)=T_{1}(x)+F_{1}(x, y), N_{2}(x, y)=T_{2}(y)+F_{2}(x, y)$.

If $\lambda$ satisfies that

$$
0<\lambda<\min \left\{\frac{\alpha_{1}-r_{2}}{t_{2} \alpha_{1}}, \frac{\alpha_{2}-s_{1}}{s_{1} \alpha_{2}}\right\}, \quad r_{2}<\alpha_{1}, s_{1}<\alpha_{2}, \frac{s_{1} r_{2}}{\alpha_{1} \alpha_{2}}<1,
$$

and $\left(f_{1}, f_{2}\right) \in E_{1} \times E_{2}$, then

(i) for any $\lambda$ in (2.1), there exists $\left(x_{\lambda}, y_{\lambda}\right) \in E_{1} \times E_{2}$ such that

$$
\left\{\begin{array}{l}
f_{1} \in J_{\lambda}^{N_{1}\left(\cdot, y_{\lambda}\right)} x_{\lambda}+M_{1}\left(h_{1}\left(x_{\lambda}\right), g_{1}\left(x_{\lambda}\right)\right), \\
f_{2} \in J_{\lambda}^{N_{2}\left(x_{\lambda}, \cdot\right)} y_{\lambda}+M_{2}\left(h_{2}\left(y_{\lambda}\right), g_{2}\left(y_{\lambda}\right)\right),
\end{array}\right.
$$

and $\left\{x_{\lambda}\right\}_{\lambda \rightarrow 0}$ and $\left\{y_{\lambda}\right\}_{\lambda \rightarrow 0}$ are bounded;

(ii) if $\left\{J_{\lambda}^{N_{1}(\cdot, y)} x_{\lambda}\right\}_{\lambda \rightarrow 0},\left\{J_{\lambda}^{N_{2}(x, \cdot)} y_{\lambda}\right\}_{\lambda \rightarrow 0}$ are bounded, then there exists unique $(x, y) \in E_{1} \times E_{2}$, which is a solution of Problem (1.1), such that $x_{\lambda} \rightarrow x, y_{\lambda} \rightarrow y$ as $\lambda \rightarrow 0$.

Remark 2.1 Equation (2.2) is called the system of Yosida approximation inclusions (equations).

Proof of Theorem 2.1 (i) By Definition 1.3, we can easily show that $\left(x_{\lambda}, y_{\lambda}\right)$ satisfies (2.2), if and only if $\left(x_{\lambda}, y_{\lambda}\right)$ satisfies the relation that

$$
\begin{aligned}
& x=R_{\lambda}^{M_{1}\left(h_{1}(\cdot), g_{1}(\cdot)\right)}\left[\lambda f_{1}+R_{\lambda}^{N_{1}(\cdot, y)} x\right] \triangleq B_{1}(x, y), \\
& y=R_{\lambda}^{M_{2}\left(h_{2}(\cdot), g_{2}(\cdot)\right)}\left[\lambda f_{2}+R_{\lambda}^{N_{2}(x, \cdot)} y\right] \triangleq B_{2}(x, y) .
\end{aligned}
$$

Now, we study the mapping $B_{i}: E_{1} \times E_{2} \rightarrow E_{i}(i=1,2)$ defined by (2.3). By Proposition 1.1(1), Lemma 1.1 and Lemma 1.2, and Eq. (2.3), for any $x_{1}, x_{2} \in E_{1}, y_{1}, y_{2} \in E_{2}$, we have that

$$
\begin{aligned}
& \left\|B_{1}\left(x_{1}, y_{1}\right)-B_{1}\left(x_{2}, y_{2}\right)\right\| \\
& \quad=\left\|R_{\lambda}^{M_{1}\left(h_{1}(\cdot), g_{1}(\cdot)\right)}\left[\lambda f_{1}+R_{\lambda}^{N_{1}\left(\cdot, y_{1}\right)} x_{1}\right]-R_{\lambda}^{M_{1}\left(h_{1}(\cdot), g_{1}(\cdot)\right)}\left[\lambda f_{1}+R_{\lambda}^{N_{1}\left(\cdot, y_{2}\right)} x_{2}\right]\right\|
\end{aligned}
$$




$$
\begin{aligned}
& \leq\left\|R_{\lambda}^{N_{1}\left(\cdot, y_{1}\right)} x_{1}-R_{\lambda}^{N_{1}\left(\cdot, y_{2}\right)} x_{2}\right\| \\
& \leq\left\|R_{\lambda}^{N_{1}\left(\cdot, y_{1}\right)} x_{1}-R_{\lambda}^{N_{1}\left(\cdot, y_{2}\right)} x_{1}\right\|+\left\|R_{\lambda}^{N_{1}\left(\cdot, y_{2}\right)} x_{1}-R_{\lambda}^{N_{1}\left(\cdot, y_{2}\right)} x_{2}\right\| \\
& \leq \lambda s_{1}\left\|y_{1}-y_{2}\right\|+\frac{1}{1+\lambda \alpha_{1}}\left\|x_{1}-x_{2}\right\| .
\end{aligned}
$$

Similarly, by Proposition 1.1(1), Lemma 1.1 and Lemma 1.2, we can prove that

$$
\left\|B_{2}\left(x_{1}, y_{1}\right)-B_{2}\left(x_{2}, y_{2}\right)\right\| \leq \lambda r_{2}\left\|x_{1}-x_{2}\right\|+\frac{1}{1+\lambda \alpha_{2}}\left\|y_{1}-y_{2}\right\| .
$$

Equations (2.4) and (2.5) imply that

$$
\left\|B_{1}\left(x_{1}, y_{1}\right)-B_{1}\left(x_{2}, y_{2}\right)\right\|+\left\|B_{2}\left(x_{1}, y_{1}\right)-B_{2}\left(x_{2}, y_{2}\right)\right\| \leq \theta_{1}\left\|x_{1}-x_{2}\right\|+\theta_{2}\left\|x_{1}-x_{2}\right\|
$$

where $\theta_{1}=\frac{1}{1+\lambda \alpha_{1}}+\lambda r_{2}, \theta_{2}=\frac{1}{1+\lambda \alpha_{2}}+\lambda s_{1}$. By (2.1), $0<\theta_{1}, \theta_{2}<1$. Therefore, by Lemma 2.1, for $\lambda$ in (2.1), there exists $\left(x_{\lambda}, y_{\lambda}\right) \in E_{1} \times E_{2}$ such that $x_{\lambda}=B_{1}\left(x_{\lambda}, y_{\lambda}\right), y_{\lambda}=B_{2}\left(x_{\lambda}, y_{\lambda}\right)$, i.e., $\left(x_{\lambda}, y_{\lambda}\right)$ satisfies (2.3), and hence (2.2) hold.

Now, we show that $\left\{x_{\lambda}\right\}_{\lambda \rightarrow 0}$ and $\left\{y_{\lambda}\right\}_{\lambda \rightarrow 0}$ are bounded. For $\left(x_{1}, y_{1}\right) \in E_{1} \times E_{2}$, and $\lambda$ in (2.1), let

$$
\begin{aligned}
& z_{\lambda} \in J_{\lambda}^{N_{1}\left(\cdot, y_{\lambda}\right)} x_{1}+M_{1}\left(h_{1}\left(x_{1}\right), g_{1}\left(x_{1}\right)\right) ; \\
& w_{\lambda} \in J_{\lambda}^{N_{2}\left(x_{\lambda}, \cdot\right)} y_{1}+M_{2}\left(h_{2}\left(y_{1}\right), g_{2}\left(y_{1}\right)\right) .
\end{aligned}
$$

Equations (2.6) plus (2.2) indicates that

$$
z_{\lambda}-J_{\lambda}^{N_{1}\left(\cdot, y_{\lambda}\right)} x_{1}-f_{1}+J_{\lambda}^{N_{1}\left(\cdot, y_{\lambda}\right)} x_{\lambda} \in M_{1}\left(h_{1}\left(x_{1}\right), g_{1}\left(x_{1}\right)\right)-M_{1}\left(h_{1}\left(x_{\lambda}\right), g_{1}\left(x_{\lambda}\right)\right)
$$

By Lemma 1.1 and condition (1) in Theorem 2.1, we obtain that

$$
\begin{aligned}
0 & \leq\left\langle z_{\lambda}-f_{1}-J_{\lambda}^{N_{1}\left(\cdot, y_{\lambda}\right)} x_{1}+J_{\lambda}^{N_{1}\left(\cdot, y_{\lambda}\right)} x_{\lambda}, j\left(x_{1}-x_{\lambda}\right)\right\rangle \\
& =\left\langle z_{\lambda}-f_{1}, j\left(x_{1}-x_{\lambda}\right)\right\rangle-\left\langle J_{\lambda}^{N_{1}\left(\cdot, y_{\lambda}\right)} x_{1}-J_{\lambda}^{N_{1}\left(\cdot, y_{\lambda}\right)} x_{\lambda}, j\left(x_{1}-x_{\lambda}\right)\right\rangle \\
& \leq\left\|z_{\lambda}-f_{1}\right\|\left\|x_{1}-x_{\lambda}\right\|-\frac{\alpha_{1}}{1+\lambda \alpha_{1}}\left\|x_{1}-x_{\lambda}\right\|^{2} .
\end{aligned}
$$

By Definition 1.3(ii), Proposition 1.1(2) and Lemma 1.2, we get that

$$
\begin{aligned}
\left\|J_{\lambda}^{N_{1}\left(\cdot, y_{\lambda}\right)} x_{1}\right\| & \leq\left\|J_{\lambda}^{N_{1}\left(\cdot, y_{\lambda}\right)} x_{1}-J_{\lambda}^{N_{1}\left(\cdot, y_{1}\right)} x_{1}\right\|+\left\|J_{\lambda}^{N_{1}\left(\cdot, y_{1}\right)} x_{1}\right\| \\
& =\left\|\frac{1}{\lambda}\left(x_{1}-R_{\lambda}^{N_{1}\left(\cdot, y_{\lambda}\right)} x_{1}-x_{1}+R_{\lambda}^{N_{1}\left(\cdot, y_{1}\right)} x_{1}\right)\right\|+\left|N_{1}\left(\cdot, y_{1}\right) x_{1}\right| \\
& \leq \frac{s_{1}}{1+\lambda \alpha_{1}}\left\|y_{1}-y_{\lambda}\right\|+\left|N_{1}\left(x_{1}, y_{1}\right)\right| .
\end{aligned}
$$

For any $\lambda$ in (2.1), take $u_{\lambda} \in M_{1}\left(h_{1}\left(x_{1}\right), g_{1}\left(x_{1}\right)\right), v_{\lambda} \in M_{2}\left(h_{2}\left(y_{1}\right), g_{2}\left(y_{1}\right)\right)$ such that $z_{\lambda}=$ $J_{\lambda}^{N_{1}\left(\cdot, y_{\lambda}\right)} x_{1}+u_{\lambda}, w_{\lambda}=J_{\lambda}^{N_{2}\left(x_{\lambda}, \cdot\right)} y_{1}+v_{\lambda}$. Since $\left\{u_{\lambda}\right\} \subset M_{1}\left(h_{1}\left(x_{1}\right), g_{1}\left(x_{1}\right)\right)$ and $\left\{v_{\lambda}\right\} \subset M_{2}\left(h_{2}\left(y_{1}\right)\right.$, $\left.g_{2}\left(y_{1}\right)\right)$, by condition (1), $\left\{u_{\lambda}\right\}$ and $\left\{v_{\lambda}\right\}$ are bounded. Combining (2.6), (2.8) and (2.9) yields 
that

$$
\begin{aligned}
\left\|x_{1}-x_{\lambda}\right\| & \leq \frac{1+\lambda \alpha_{1}}{\alpha_{1}}\left\|z_{\lambda}-f_{1}\right\| \leq \frac{1+\lambda \alpha_{1}}{\alpha_{1}}\left(\left\|z_{\lambda}\right\|+\left\|f_{1}\right\|\right) \\
& \leq \frac{1+\lambda \alpha_{1}}{\alpha_{1}}\left(\left\|J_{\lambda}^{N_{1}\left(\cdot, y_{\lambda}\right)} x_{1}\right\|+\left\|u_{\lambda}\right\|+\left\|f_{1}\right\|\right) \\
& \leq \frac{1+\lambda \alpha_{1}}{\alpha_{1}}\left(\frac{s_{1}}{1+\lambda \alpha_{1}}\left\|y_{1}-y_{\lambda}\right\|+\left|N_{1}\left(x_{1}, y_{1}\right)\right|+\left\|u_{\lambda}\right\|+\left\|f_{1}\right\|\right) .
\end{aligned}
$$

By using similar methods, we obtain that

$$
\left\|y_{1}-y_{\lambda}\right\| \leq \frac{1+\lambda \alpha_{2}}{\alpha_{2}}\left(\frac{r_{2}}{1+\lambda \alpha_{2}}\left\|x_{1}-x_{\lambda}\right\|+\left|N_{2}\left(x_{1}, y_{1}\right)\right|+\left\|v_{\lambda}\right\|+\left\|f_{2}\right\|\right)
$$

It follows from (2.10) and (2.11) that $\left\{x_{\lambda}\right\}_{\lambda \rightarrow 0}$ and $\left\{y_{\lambda}\right\}_{\lambda \rightarrow 0}$ are bounded since $0<\frac{s_{1} r_{2}}{\alpha_{1} \alpha_{2}}<1$.

(ii) Note that for $\lambda, \mu>0$

$$
f_{1}-J_{\lambda}^{N_{1}\left(\cdot, y_{\lambda}\right)} x_{\lambda} \in M_{1}\left(h_{1}(\cdot), g_{1}(\cdot)\right) x_{\lambda} \quad \text { and } \quad f_{1}-J_{\mu}^{N_{1}\left(\cdot, y_{\mu}\right)} x_{\mu} \in M_{1}\left(h_{1}(\cdot), g_{1}(\cdot)\right) x_{\mu}
$$

By Proposition 1.1(4), we have that

$$
\begin{aligned}
0 \leq & \left\langle-J_{\lambda}^{N_{1}\left(\cdot, y_{\lambda}\right)} x_{\lambda}+J_{\mu}^{N_{1}\left(\cdot, y_{\mu}\right)} x_{\mu}, j\left(x_{\lambda}-x_{u}\right)\right\rangle \\
= & \left\langle J_{\lambda}^{N_{1}\left(\cdot, y_{\lambda}\right)} x_{\lambda}-J_{\mu}^{N_{1}\left(\cdot, y_{\mu}\right)} x_{\mu}, j\left(R_{\lambda}^{N_{1}\left(\cdot, y_{\lambda}\right)} x_{\lambda}-R_{\mu}^{N_{1}\left(\cdot, y_{\mu}\right)} x_{\mu}\right)-j\left(x_{\lambda}-x_{\mu}\right)\right\rangle \\
& -\left\langle J_{\lambda}^{N_{1}\left(\cdot, y_{\lambda}\right)} x_{\lambda}-J_{\mu}^{N_{1}\left(\cdot, y_{\mu}\right)} x_{\mu}, j\left(R_{\lambda}^{N_{1}\left(\cdot, y_{\lambda}\right)} x_{\lambda}-R_{\mu}^{N_{1}\left(\cdot, y_{\mu}\right)} x_{\mu}\right)\right\rangle \\
\leq & \left\langle J_{\lambda}^{N_{1}\left(\cdot, y_{\lambda}\right)} x_{\lambda}-J_{\mu}^{N_{1}\left(\cdot, y_{\mu}\right)} x_{\mu}, j\left(R_{\lambda}^{N_{1}\left(\cdot, y_{\lambda}\right)} x_{\lambda}-R_{\mu}^{N_{1}\left(\cdot, y_{\mu}\right)} x_{\mu}\right)-j\left(x_{\lambda}-x_{\mu}\right)\right\rangle \\
& -\alpha_{1}\left\|R_{\lambda}^{N_{1}\left(\cdot, y_{\lambda}\right)} x_{\lambda}-R_{\mu}^{N_{1}\left(\cdot, y_{\mu}\right)} x_{\mu}\right\|^{2},
\end{aligned}
$$

and hence,

$$
\begin{aligned}
& \alpha_{1}\left\|R_{\lambda}^{N_{1}\left(\cdot, y_{\lambda}\right)} x_{\lambda}-R_{\mu}^{N_{1}\left(\cdot, y_{\mu}\right)} x_{\mu}\right\|^{2} \\
& \quad \leq\left\langle J_{\lambda}^{N_{1}\left(\cdot, y_{\lambda}\right)} x_{\lambda}-J_{\mu}^{N_{1}\left(\cdot, y_{\mu}\right)} x_{\mu}, j\left(R_{\lambda}^{N_{1}\left(\cdot, y_{\lambda}\right)} x_{\lambda}-R_{\mu}^{N_{1}\left(\cdot, y_{\mu}\right)} x_{\mu}\right)-j\left(x_{\lambda}-x_{\mu}\right)\right\rangle .
\end{aligned}
$$

Since $x_{\lambda}-R_{\lambda}^{N_{1}\left(\cdot, y_{\lambda}\right)} x_{\lambda}=\lambda J_{\lambda}^{N_{1}\left(\cdot, y_{\lambda}\right)} x_{\lambda} \rightarrow 0$ (as $\left.\lambda \rightarrow 0\right),\left\{J_{\lambda}^{N_{1}\left(\cdot, y_{\lambda}\right)} x_{\lambda}\right\}_{\lambda \rightarrow 0}$ is bounded. The $j$ is uniformly continuous on bounded set, and (2.12) reduces to that

$$
\begin{aligned}
& \alpha_{1}\left\|R_{\lambda}^{N_{1}\left(\cdot, y_{\lambda}\right)} x_{\lambda}-R_{\mu}^{N_{1}\left(\cdot, y_{\mu}\right)} x_{\mu}\right\|^{2} \\
& \quad \leq O\left(\left\|x_{\lambda}-x_{\mu}-R_{\lambda}^{N_{1}\left(\cdot, y_{\lambda}\right)} x_{\lambda}+R_{\mu}^{N_{1}\left(\cdot, y_{\mu}\right)} x_{\mu}\right\|\right) \leq O(\lambda+\mu) .
\end{aligned}
$$

Similarly, we have that

$$
\alpha_{2}\left\|R_{\lambda}^{N_{2}\left(x_{\lambda}, \cdot\right)} y_{\lambda}-R_{\mu}^{N_{2}\left(x_{\mu}, \cdot\right)} y_{\mu}\right\| \leq O(\lambda+\mu)
$$

Consequently, $\left\{R_{\lambda}^{N_{1}\left(\cdot, y_{\lambda}\right)} x_{\lambda}\right\}_{\lambda \rightarrow 0}$ and $\left\{R_{\lambda}^{N_{2}\left(x_{\lambda}, \cdot\right)} y_{\lambda}\right\}_{\lambda \rightarrow 0}$ are the Cauchy net. There exists $(x, y) \in$ $E_{1} \times E_{2}$ such that $R_{\lambda}^{N_{1}\left(\cdot, y_{\lambda}\right)} x_{\lambda} \rightarrow x, R_{\lambda}^{N_{2}\left(x_{\lambda}, \cdot\right)} y_{\lambda} \rightarrow y$ as $\lambda \rightarrow 0$ from which and $x_{\lambda}-R_{\lambda}^{N_{1}\left(\cdot, y_{\lambda}\right)} x_{\lambda}=$ $\lambda J_{\lambda}^{N_{1}\left(\cdot, y_{\lambda}\right)} x_{\lambda}$ and $y_{\lambda}-R_{\lambda}^{N_{2}\left(x_{\lambda}, \cdot\right)} y_{\lambda}=\lambda J_{\lambda}^{N_{2}\left(x_{\lambda}, \cdot\right)} y_{\lambda}$, it follows that $x_{\lambda} \rightarrow x$ and $y_{\lambda} \rightarrow y$ as $\lambda \rightarrow 0$. 
Now, we show that $(x, y)$ is a solution of (1.1). Since $E_{i}$ is reflexive and $\left\{J_{\lambda}^{N_{1}\left(\cdot, y_{\lambda}\right)} x_{\lambda}\right\}_{\lambda \rightarrow 0}$ and $\left\{J_{\lambda}^{N_{2}\left(x_{\lambda}, \cdot\right)} y_{\lambda}\right\}_{\lambda \rightarrow 0}$ are bounded, there exist $\lambda_{n}>0(n=1,2, \ldots)$ such that $\lambda_{n} \rightarrow 0$ and $J_{\lambda_{n}}^{N_{1}\left(\cdot, y_{\lambda_{n}}\right)} x_{\lambda_{n}} \rightarrow z_{1}, J_{\lambda_{n}}^{N_{2}\left(x_{\lambda_{n}}, \cdot\right)} y_{\lambda_{n}} \rightarrow z_{2}$ for some $\left(z_{1}, z_{2}\right) \in E_{1} \times E_{2}$. Let $w_{\lambda_{n}}^{\prime}=f_{1}-J_{\lambda_{n}}^{N_{1}\left(\cdot, y_{\lambda_{n}}\right)} x_{\lambda_{n}} \in$ $M_{1}\left(h_{1}\left(x_{\lambda_{n}}\right), g_{1}\left(x_{\lambda_{n}}\right)\right), w_{\lambda_{n}}^{\prime \prime}=f_{2}-J_{\lambda_{n}}^{N_{2}\left(x_{\lambda_{n}},\right)} y_{\lambda_{n}} \in M_{2}\left(h_{2}\left(x_{\lambda_{n}}\right), g_{2}\left(y_{\lambda_{n}}\right)\right)$. Then $w_{\lambda_{n}}^{\prime} \rightarrow w_{1}, w_{\lambda_{n}}^{\prime \prime} \rightarrow$ $w_{2}$ for some $\left(w_{1}, w_{2}\right) \in E_{1} \times E_{2}$. Since $N_{1}(\cdot, y), N_{2}(x, \cdot)\left(\forall(x, y) \in E_{1} \times E_{2}\right), T_{i}$ and $M_{i}\left(h_{i}(\cdot)\right.$, $\left.g_{1}(\cdot)\right)(i=1,2)$ are demiclosed (see Proposition 1.1(5)), we have that

$$
\begin{aligned}
& x \in E_{1}=D\left(N_{1}(\cdot, y)\right) \cap D\left(M_{1}\left(h_{1}(\cdot), g_{1}(\cdot)\right)\right), \\
& y \in E_{2}=D\left(N_{2}(x, \cdot)\right) \cap D\left(M_{2}\left(h_{2}(\cdot), g_{2}(\cdot)\right)\right), \\
& z_{1} \in N_{1}(\cdot, y) x=N_{1}(x, y)=T_{1} x+F_{1}(x, y), \\
& z_{2} \in N_{2}(x, \cdot) y=N_{2}(x, y)=T_{2} y+F_{2}(x, y), \\
& w_{1} \in M_{1}\left(h_{1}(x), g_{1}(x)\right) \text { and } w_{2} \in M_{2}\left(h_{2}(y), g_{2}(y)\right) .
\end{aligned}
$$

Therefore,

$$
\begin{aligned}
& f_{1}=z_{1}+w_{1} \in T_{1} x+F_{1}(x, y)+M_{1}\left(h_{1}(x), g_{1}(x)\right) \\
& f_{2}=z_{2}+w_{2} \in T_{2} y+F_{2}(x, y)+M_{2}\left(h_{2}(y), g_{2}(y)\right) .
\end{aligned}
$$

Finally, we show the uniqueness of solutions. Let $(x, y)$ and $\left(x_{1}, y_{1}\right)$ be two solutions of Problem (1.1). Let $u \in T_{1} x, u_{1} \in T_{1} x_{1}, w \in M_{1}\left(h_{1}(x), g_{1}(x)\right), w_{1} \in M_{1}\left(h_{1}\left(x_{1}\right), g_{1}\left(x_{1}\right)\right)$ such that

$$
f_{1}=u+F_{1}(x, y)+w, \quad f_{1}=u_{1}+F_{1}\left(x_{1}, y_{1}\right)+w_{1}
$$

Then by accretiveness of $M_{i}$ and $T_{i}$, we have that

$$
\begin{aligned}
0 & =\left\langle f_{1}-f_{1}, j\left(x-x_{1}\right)\right\rangle \\
& =\left\langle u+F_{1}(x, y)+w-u_{1}-F_{1}\left(x_{1}, y_{1}\right)-w_{1}, j\left(x-x_{1}\right)\right\rangle \\
& \geq\left\langle F_{1}(x, y)-F_{1}\left(x_{1}, y_{1}\right), j\left(x-x_{1}\right)\right\rangle \\
& =\left\langle F_{1}(x, y)-F_{1}\left(x_{1}, y\right), j\left(x-x_{1}\right)\right\rangle+\left\langle F_{1}\left(x_{1}, y\right)-F_{1}\left(x_{1}, y_{1}\right), j\left(x-x_{1}\right)\right\rangle \\
& \geq \alpha_{1}\left\|x-x_{1}\right\|^{2}-\left\|F_{1}\left(x_{1}, y\right)-F_{1}\left(x_{1}, y_{1}\right)\right\|\left\|x-x_{1}\right\| \\
& \geq \alpha_{1}\left\|x-x_{1}\right\|^{2}-s_{1}\left\|y-y_{1}\right\|\left\|x-x_{1}\right\| .
\end{aligned}
$$

That is,

$$
\left\|x-x_{1}\right\| \leq \frac{s_{1}}{\alpha_{1}}\left\|y-y_{1}\right\|
$$

Let $v \in T_{2} y, v_{1} \in T_{2} y_{1}, z \in M_{2}\left(h_{2}(y), g_{2}(y)\right), z_{1} \in M_{2}\left(h_{2}\left(y_{1}\right), g_{2}\left(y_{1}\right)\right)$ such that

$$
\begin{aligned}
& f_{2}=v+F_{2}(x, y)+w \\
& f_{2}=v_{1}+F_{2}\left(x_{1}, y_{1}\right)+w_{1} .
\end{aligned}
$$


The by the similar discussion, we have that

$$
\left\|y-y_{1}\right\| \leq \frac{r_{2}}{\alpha_{2}}\left\|x-x_{1}\right\|
$$

Equations (2.1), (2.13) and (2.14) imply that $x=x_{1}, y=y_{1}$.

Theorem 2.2 Suppose that $E_{i}, T_{i}, M_{i}, F_{i}, f_{i}$ and $h_{i}(i=1,2)$ are the same as in Theorem 2.1. Iffor any $R_{i}>0$, there exist $L_{i}>0, a_{i}>0$ and $0<L_{i}<1$ such that

$$
\begin{aligned}
& \left|T_{1} x\right| \leq L_{1}\left|M_{1}\left(h_{1}(x), g_{1}(x)\right)\right|+a_{1}, \quad\|x\| \leq R_{1}, \\
& \left|T_{2} y\right| \leq L_{2}\left|M_{2}\left(h_{2}(y), g_{2}(y)\right)\right|+a_{2}, \quad\|y\| \leq R_{2},
\end{aligned}
$$

then Problem (1.1) has a unique solution.

Proof It suffices to show that $\left\{J_{\lambda}^{N_{1}\left(\cdot, y_{\lambda}\right)} x_{\lambda}\right\}_{\lambda \rightarrow 0}$ and $\left\{J_{\lambda}^{N_{2}\left(x_{\lambda}, \cdot\right)} y_{\lambda}\right\}_{\lambda \rightarrow 0}$ in Theorem 2.1 are bounded. Because $\left\{x_{\lambda}\right\}$ and $\left\{y_{\lambda}\right\}$ are bounded, therefore, there exists $R_{i}>0(i=1,2)$ such that for $\lambda$ in (2.1), $\left\|x_{\lambda}\right\| \leq R_{1}$ and $\left\|y_{\lambda}\right\| \leq R_{2}$. By Proposition 1.1(2) and (2.15),

$$
\begin{aligned}
\left\|J_{\lambda}^{N_{1}\left(\cdot, y_{\lambda}\right)} x_{\lambda}\right\| & \leq\left|N_{1}\left(x_{\lambda}, y_{\lambda}\right)\right|=\left|T_{1} x_{\lambda}+F_{1}\left(x_{\lambda}, y_{\lambda}\right)\right| \\
& =\inf \left\{\|u\|: u=u_{0}+F_{1}\left(x_{\lambda}, y_{\lambda}\right) \in T_{1} x_{\lambda}+F_{1}\left(x_{\lambda}, y_{\lambda}\right)\right\} \\
& =\inf \left\{\left\|u_{0}+F_{1}\left(x_{\lambda}, y_{\lambda}\right)\right\|: u_{0} \in T_{1} x_{\lambda}\right\} \\
& \leq \inf \left\{\left\|u_{0}\right\|: u_{0} \in T_{1} x_{\lambda}\right\}+\left\|F_{1}\left(x_{\lambda}, y_{\lambda}\right)\right\| \\
& =\left|T_{1} x_{\lambda}\right|+\left\|F_{1}\left(x_{\lambda}, y_{\lambda}\right)\right\| \\
& \leq L_{1}\left|M_{1}\left(h_{1}\left(x_{\lambda}\right), g_{1}\left(x_{\lambda}\right)\right)\right|+\left\|F_{1}\left(x_{\lambda}, y_{\lambda}\right)\right\|+a_{1} ;
\end{aligned}
$$

Similarly, by Proposition 1.1(2) and (2.16), we get that

$$
\left\|J_{\lambda}^{N_{2}\left(x_{\lambda}, \cdot\right)} y_{\lambda}\right\| \leq L_{2}\left|M_{2}\left(h_{2}\left(y_{\lambda}\right), g_{2}\left(y_{\lambda}\right)\right)\right|+\left\|F_{2}\left(x_{\lambda}, y_{\lambda}\right)\right\|+a_{2} .
$$

By (2.2),

$$
\begin{aligned}
& \left|M_{1}\left(h_{1}\left(x_{\lambda}\right), g_{1}\left(x_{\lambda}\right)\right)\right| \leq\left\|f_{1}\right\|+\left\|J_{\lambda}^{N_{1}\left(\cdot, y_{\lambda}\right)} x_{\lambda}\right\|, \\
& \left|M_{2}\left(h_{2}\left(y_{\lambda}\right), g_{2}\left(y_{\lambda}\right)\right)\right| \leq\left\|f_{2}\right\|+\left\|J_{\lambda}^{N_{2}\left(x_{\lambda}, \cdot\right)} y_{\lambda}\right\| .
\end{aligned}
$$

Therefore, from (2.17)-(2.20), it follows that

$$
\begin{aligned}
& \left\|J_{\lambda}^{N_{1}\left(\cdot, y_{\lambda}\right)} x_{\lambda}\right\| \leq \frac{L_{1}}{1-L_{1}}\left\|f_{1}\right\|+\frac{1}{1-L_{1}}\left\|F_{1}\left(x_{\lambda}, y_{\lambda}\right)\right\|+\frac{a_{1}}{1-L_{1}}, \\
& \left\|J_{\lambda}^{\left.N_{2}\left(x_{\lambda},\right)\right)} y_{\lambda}\right\| \leq \frac{L_{2}}{1-L_{2}}\left\|f_{2}\right\|+\frac{1}{1-L_{2}}\left\|F_{2}\left(x_{\lambda}, y_{\lambda}\right)\right\|+\frac{a_{2}}{1-L_{2}} .
\end{aligned}
$$

Since $F_{i}(i=1,2)$ is uniformly continuous, $F_{i}$ map bounded set in $E_{1} \times E_{2}$ to bounded set. Hence, (2.21) and (2.22) imply that $\left\{J_{\lambda}^{N_{1}\left(\cdot, y_{\lambda}\right)} x_{\lambda}\right\}_{\lambda \rightarrow 0}$ and $\left\{J_{\lambda}^{N_{2}\left(x_{\lambda}, \cdot\right)} y_{\lambda}\right\}_{\lambda \rightarrow 0}$ are bounded. $\square$ 
Theorem 2.3 Suppose that $E_{i}, T_{i}, M_{i}, h_{i}, g_{i}, F_{i}$ and $f_{i}(i=1,2)$ are the same as in Theorem 2.1. If for any $R_{i}>0$, there exists bounded functional $B_{i}: E_{1} \times E_{2} \rightarrow \Re_{+}$(i.e., $B_{i}$ map a bounded set in $E_{1} \times E_{2}$ to a bounded set in $\left.\Re_{+}\right)$such that for $[x, z] \in \operatorname{Graph}\left(M_{1}\left(h_{1}(\cdot), g_{1}(\cdot)\right)\right)$, $[y, w] \in \operatorname{Graph}\left(M_{2}\left(h_{2}(\cdot), g_{2}(\cdot)\right)\right)$ and $\lambda>0$,

$$
\begin{aligned}
& \left\langle z, j J_{\lambda}^{N_{1}(\cdot, y)} x\right\rangle \geq-B_{1}(x, y), \\
& \left\langle w, j J_{\lambda}^{N_{2}(x, \cdot)} y\right\rangle \geq-B_{2}(x, y), \quad\|x\| \leq R_{1},\|y\| \leq R_{2}
\end{aligned}
$$

for $x \in E_{1},\|x\| \leq R_{1}, y \in E,\|y\| \leq R_{2}$, then Problem (1.1) has a unique solution.

Proof It suffices to show that $\left\{J_{\lambda}^{N_{1}\left(\cdot, y_{\lambda}\right)} x_{\lambda}\right\}_{\lambda \rightarrow 0}$ and $\left\{J_{\lambda}^{N_{2}\left(x_{\lambda}, \cdot\right)} y_{\lambda}\right\}_{\lambda \rightarrow 0}$ are bounded. Since $\left\{x_{\lambda}\right\}_{\lambda \rightarrow 0}$ and $\left\{y_{\lambda}\right\}_{\lambda \rightarrow 0}$ are bounded, then by (2.23), for $u_{\lambda} \in M_{1}\left(h_{1}(\cdot), g_{2}(\cdot)\right) x_{\lambda}$,

$$
\begin{aligned}
\left\|f_{1}\right\|\left\|J_{\lambda}^{N_{1}\left(\cdot, y_{\lambda}\right)} x_{\lambda}\right\| & \geq\left\langle f_{1}, j J_{\lambda}^{N_{1}\left(\cdot, y_{\lambda}\right)} x_{\lambda}\right\rangle=\left\langle J_{\lambda}^{N_{1}\left(\cdot, y_{\lambda}\right)} x_{\lambda}+u_{\lambda}, j J_{\lambda}^{N_{1}\left(\cdot, y_{\lambda}\right)} x_{\lambda}\right\rangle \\
& =\left\|J_{\lambda}^{N_{1}\left(\cdot, y_{\lambda}\right)} x_{\lambda}\right\|^{2}+\left\langle u_{\lambda}, j J_{\lambda}^{N_{1}\left(\cdot, y_{\lambda}\right)} x_{\lambda}\right\rangle \geq\left\|J_{\lambda}^{N_{1}\left(\cdot, y_{\lambda}\right)} x_{\lambda}\right\|^{2}-B_{1}\left(x_{\lambda}, y_{\lambda}\right),
\end{aligned}
$$

which implies that $\left\|J_{\lambda}^{N_{1}\left(\cdot, y_{\lambda}\right)} x_{\lambda}\right\| \leq\left(B_{1}\left(x_{\lambda}, y_{\lambda}\right)+\frac{1}{4}\left\|f_{1}\right\|^{2}\right)^{\frac{1}{2}}+\frac{\left\|f_{1}\right\|}{2}$. Similarly, $\left\|J_{\lambda}^{N_{2}\left(x_{\lambda}, \cdot\right)} y_{\lambda}\right\| \leq$ $\left(B_{2}\left(x_{\lambda}, y_{\lambda}\right)\right)^{\frac{1}{2}}+\frac{1}{2}\left\|f_{2}\right\|$. This completes the proof of Theorem 2.3.

\section{Conclusion and future perspective}

Two of the most difficult and important problems in variation inclusions are the establishment of system of variational inclusions and the development of an efficient numerical methods. A new system of generalized variational inclusions in the Banach space under the assumption with no continuousness is introduced, and some existence and uniqueness theorems of solutions for this kind of system of generalized variational inclusions are proved by using the Yosida approximation technique for $\mathrm{m}$-accretive operator.

More approaches [13-15], which have been applied in variational inequalities, could be manipulated in variational inclusions. We will make further research to solve this kind of system of generalized variational inclusions by using extragradient method and implicit iterative methods.

\footnotetext{
Competing interests

The author declares that they have no competing interests.
}

\section{Acknowledgements}

The author thanks for the guidance and support my supervisor Prof. Li-Wei Liu who taught at the department of mathematics in Nanchang university. The author thanks the anonymous referees for reading this paper carefully, providing valuable suggestions and comments. The work was supported by the National Science Foundation of China (No. 10561007) and the Youth Science Foundation of Jiangxi Provincial Department of Science and Technology (No. 20122BAB211021).

Received: 18 May 2013 Accepted: 26 September 2013 Published: 07 Nov 2013

\section{References}

1. Kazmi, KR, Khan, FA, Shahzad, M: A system of generalized variational inclusions involving generalized $H(\cdot, \cdot)$-accretive mapping in real uniformly smooth Banach spaces. Appl. Math. Comput. 217, 9679-9688 (2011)

2. Kazmi, KR, Khan, FA: Iterative approximation of a unique solution of a system of variational-like inclusions in real q-uniformly smooth Banach spaces. Nonlinear Anal. 67, 917-929 (2007)

3. Verma, RU: General system of A-monotone nonlinear variational inclusion problems with applications. J. Optim. Theory Appl. 131(1), 151-157 (2006)

4. Verma, RU: General nonlinear variational inclusions problems involving A-monotone mapping. Appl. Math. Lett. 19, 960-963 (2006) 
5. Fang, YP, Huang, NJ: Iterative algorithm for a system of variational inclusions involving $\mathrm{H}$-accretive operators in Banach spaces. Acta Math. Hung. 108(3), 183-195 (2005)

6. Zeng, LC, Guo, SM, Yao, JC: Characterization of $\mathrm{H}$-monotone operators with applications to variational inclusions. Comput. Math. Appl. 50, 329-337 (2005)

7. Noor, MA, Huang, Z: Some resolvent iterative methods for variational inclusion and nonexpansive mappings. Appl. Math. Comput. 194, 267-275 (2007)

8. Barbu, V: Nonlinear Semigroups and Differential Equations in Banach Spaces. International publishing, Leyden (1976)

9. Browder, FE: Nonlinear Operator and Nonlinear Equations of Evolution in Banach Spaces. Am. Math. Soc., Providence (1976)

10. Lakshmikantham, V, Leela, S: Nonlinear Differential Equations in Abstract Spaces. Pergamon Press, Oxford (1981)

11. Kobayashi, Y: Difference approximation of Cauchy problems for quasi-dissipative operators and generation of nonlinear semigroups. J. Math. Soc. Jpn. 27, 640-665 (1975)

12. Cao, HW: Sensitivity analysis for a system of generalized nonlinear mixed quasi-variational inclusions with H-monotone operators. J. Appl. Math. (2011). doi:10.1155/2011/921835

13. Yao, Y, Noor, MA, Liou, YC: Strong convergence of a modified extra-gradient method to the minimum-norm solution of variational inequalities. Abstr. Appl. Anal. (2012). doi:10.1155/2012/817436

14. Yao, Y, Liou, YC, Li, CL, Lin, HT: Extended extra-gradient methods for generalized variational inequalities. J. Appl. Math. (2012). doi:10.1155/2012/237083

15. Noor, MA, Noor, Kl, Huang, Z, Al-said, E: Implicit schemes for solving extended general nonconvex variational inequalities. J. Appl. Math. (2012). doi:10.1155/2012/646259

10.1186/1029-242X-2013-455

Cite this article as: Cao: Yosida approximation equations technique for system of generalized set-valued variational inclusions. Journal of Inequalities and Applications 2013, 2013:455

\section{Submit your manuscript to a SpringerOpen ${ }^{\circ}$ journal and benefit from:}

- Convenient online submission

Rigorous peer review

- Immediate publication on acceptance

- Open access: articles freely available online

- High visibility within the field

- Retaining the copyright to your article 